\title{
Article
}

\section{Existence of solution for a nonlinear fifth-order three-point boundary value problem}

\section{Zouaoui Bekri ${ }^{1, *}$ and Slimane Benaicha ${ }^{2}$}

1 Laboratory of fundamental and applied mathematics, University of Oran 1, Ahmed Ben Bella, Es-senia, 31000 Oran, Algeria.

2 Laboratory of fundamental and applied mathematics, University of Oran 1, Ahmed Ben Bella, Es-senia, 31000 Oran, Algeria.; slimanebenaicha@yahoo.fr

* Correspondence: zouaouibekri@yahoo.fr

Received: 2 September 2019; Accepted: 22 October 2019; Published: 31 December 2019.

Abstract: In this paper, we explore the existence of nontrivial solution for the fifth-order three-point boundary value problem of the form $u^{(5)}(t)+f(t, u(t))=0, \quad 0<t<1$, with boundary conditions $u(0)=0, \quad u^{\prime}(0)=$ $u^{\prime \prime}(0)=u^{\prime \prime \prime}(0)=0, u(1)=\alpha u(\eta)$, where $0<\eta<1, \alpha \in \mathbb{R}, \alpha \eta^{4} \neq 1, f \in C([0,1] \times \mathbb{R}, \mathbb{R})$. Under certain growth conditions on the non-linearity $f$ and using Leray-Schauder nonlinear alternative, we prove the existence of at least one solution of the posed problem. Furthermore, the obtained results are further illustrated by mean of some examples.

Keywords: Implicit fractional differential equation, Caputo-Hadamard fractional derivative, fixed point theorems, existence, uniqueness.

MSC: 34B10, 34B15, 34K10.

\section{Introduction}

$\mathbf{T}$ he study of fourth-order three-point boundary value problems (BVP) for ordinary differential equations arise in a variety of different areas of applied mathematics and physics. Various authors studied the existence of positive solutions for $n$ th-order $m$-point boundary value problems using different methods, for example, fixed point theorems in cones, nonlinear alternative of Leray-Schauder, and Krasnoselskii's fixed point theorem, see [1-5] and the references therein.

In 2003, by using the Leray-Schauder degree theory, Liu and Ge [6] proved the existence of positive solutions for $(n-1,1)$ three-point boundary value problems with coefficient that changes sign given as follows:

$$
\begin{gathered}
u^{(n)}(t)+\lambda a(t) f(u(t))=0, \quad \mathrm{t} \in(0,1), \\
u(0)=\alpha u(\eta), \quad u(1)=\beta u(\eta), \quad u^{(i)}(0)=0 \text { for } i=1,2, \ldots, n-2, \\
\text { and } u^{(n-2)}(0)=\alpha u^{(n-2)}(\eta), \quad u^{(n-2)}(1)=\beta u^{(n-2)}(\eta), \quad u^{(i)}(0)=0 \text { for } i=1,2, . ., n-3,
\end{gathered}
$$

where $\eta \in(0,1), \alpha \geq 0, \beta \geq 0$, and $a:(0,1) \rightarrow \mathbb{R}$ may change sign and $\mathbb{R}=(-\infty, \infty), f(0)>0, \lambda>0$ is a parameter.

In 2005, Eloea and Ahmad [7] studied the existence of positive solutions of a nonlinear $n$ th-order boundary value problem with nonlocal conditions as follows:

$$
\begin{gathered}
u^{(n)}(t)+a(t) f(u(t))=0, \quad \mathrm{t} \in(0,1), \\
u(0)=0, \quad u^{\prime}(0)=0, \ldots, u^{(n-2)}(0)=0, \quad \alpha u(\eta)=u(1),
\end{gathered}
$$

where $0<\eta<1,0<\alpha \eta^{n-1}<1, f$ is either superlinear or sublinear. 
In 2009, Bai et al. [8] used fixed-point theory to study the existence of positive solutions of a singular nth-order three-point boundary value problem on time scales represented as:

$$
\begin{gathered}
u^{n}(t)+a(t) f(u(t))=0, \quad \mathrm{t} \in(0,1), \\
u(a)=\alpha u(\eta), \quad u^{\prime}(a)=0, \ldots, u^{(n-2)}(a)=0, \quad u(b)=\beta u(\eta),
\end{gathered}
$$

where $a<\eta<b, 0 \leq a<1,0<\beta(\eta-a)^{n-1}<(1-\alpha)(b-a)^{n-1}+\alpha(\eta-a)^{n-1}, f \in C([a, b] \times[0, \infty),[0, \infty))$ and $h \in C([a, b],[0, \infty))$ may be singular at $t=a$ and $t=b$.

In 2004, Sun [9] studied the existence of nontrivial solution for the three-point boundary value problem:

$$
\begin{gathered}
u^{\prime \prime}(t)+f(t, u(t))=0, \quad 0 \leq t \leq 1, \\
u^{\prime}(0)=0, \quad u(1)=\alpha u^{\prime}(\eta)
\end{gathered}
$$

where $\eta \in(0,1), \alpha \in \mathbb{R}, f \in C([0,1] \times \mathbb{R}, \mathbb{R})$. The same author in [10], studied solvability of a nonlinear second-order three-point boundary value problem:

$$
\begin{gathered}
u^{\prime \prime}(t)+f(t, u(t))=0, \quad 0 \leq t \leq 1 \\
u^{\prime}(0)=0, \quad u(1)=\alpha u(\eta)
\end{gathered}
$$

where $\eta \in(0,1), \alpha \in \mathbb{R}, \alpha \neq 0, f \in C([0,1] \times \mathbb{R}, \mathbb{R})$.

$\mathrm{Li}$ and Sun [11], also used the same method to study nontrivial solution of a nonlinear second-order three-point boundary value problem:

$$
\begin{gathered}
u^{\prime \prime}(t)+f(t, u(t))=0, \quad 0 \leq t \leq 1, \\
a u(0)-b u^{\prime}(0)=0, \quad u(1)-\alpha u(\eta)=0,
\end{gathered}
$$

where $\eta \in(0,1), a, b, \alpha \in \mathbb{R}$, with $a^{2}+b^{2}>0$.

Motivated by the above work, we extend the results obtained for second-order boundary value problem to fourth-order boundary value problem using a different method from [7]. We prove the existence of nontrivial solution for the fourth-order three-point boundary value problem (BVP):

$$
\begin{gathered}
u^{(5)}(t)+f(t, u(t))=0, \quad 0<t<1 . \\
u(0)=0, \quad u^{\prime}(0)=u^{\prime \prime}(0)=u^{\prime \prime \prime}(0)=0, \quad u(1)=\alpha u(\eta),
\end{gathered}
$$

where $0<\eta<1, \alpha \in \mathbb{R}, \alpha \eta^{4} \neq 1, f \in C([0,1] \times \mathbb{R}, \mathbb{R}), \mathbb{R}=(-\infty, \infty)$.

This paper is organized as follows: in Section 2, we present two lemmas that will be helpful in proving our main results, in Section 3, we present our main results and finally, in Section 4, we illustrated our results with examples.

\section{Preliminaries}

Let $E=C[0,1]$ with the norm $\|y\|=\sup _{t \in[0,1]}|y(t)|$ for any $u \in E$. A solution $u(t)$ of the BVP (1)-(2) is called nontrivial solution if $u(t) \neq 0$. To get our results, we need to the following lemma.

Lemma 1. Let $y \in C([0,1]), \alpha \eta^{4} \neq 1$, then the boundary value problem

$$
\begin{gathered}
u^{(5)}(t)+y(t)=0, \quad 0<t<1, \\
u(0)=0, \quad u^{\prime}(0)=u^{\prime \prime}(0)=u^{\prime \prime \prime}(0)=0, \quad u(1)=\alpha u(\eta),
\end{gathered}
$$

has a unique solution

$$
u(t)=-\frac{1}{24} \int_{0}^{t}(t-s)^{4} y(s) d s+\frac{t^{4}}{24\left(1-\alpha \eta^{4}\right)} \int_{0}^{1}(1-s)^{4} y(s) d s-\frac{\alpha t^{4}}{24\left(1-\alpha \eta^{4}\right)} \int_{0}^{\eta}(\eta-s)^{4} y(s) d s
$$


Proof. Rewriting the differential equation as $u^{(5)}(t)=-y(t)$, and integrating five times from 0 to 1 , we obtain

$$
u(t)=-\frac{1}{24} \int_{0}^{t}(t-s)^{4} y(s) d s+\frac{t^{4}}{24} c+\frac{t^{3}}{6} c_{1}+\frac{t^{2}}{2} c_{2}+t c_{3}+c_{4}
$$

By the boundary conditions (2), we have

$$
u(0)=0, \quad u^{\prime}(0)=u^{\prime \prime}(0)=u^{\prime \prime \prime}(0)=0 \text {, i.e. } c_{1}=c_{2}=c_{3}=c_{4}=0,
$$

and $u(1)=\alpha u(\eta)$, we get

$$
c=\frac{1}{\left(1-\alpha \eta^{4}\right)} \int_{0}^{1}(1-s)^{4} y(s) d s-\frac{\alpha}{\left(1-\alpha \eta^{4}\right)} \int_{0}^{\eta}(\eta-s)^{4} y(s) d s .
$$

Compensate Equation (3) in the Equation (4), we obtain

$$
u(t)=-\frac{1}{24} \int_{0}^{t}(t-s)^{4} y(s) d s+\frac{t^{4}}{24\left(1-\alpha \eta^{4}\right)} \int_{0}^{1}(1-s)^{4} y(s) d s-\frac{\alpha t^{4}}{24\left(1-\alpha \eta^{4}\right)} \int_{0}^{\eta}(\eta-s)^{4} y(s) d s .
$$

This completes the proof.

Define the integral operator $T: E \longrightarrow E$, by

$T u(t)=-\frac{1}{24} \int_{0}^{t}(t-s)^{4} f(s, u(s)) d s+\frac{t^{4}}{24\left(1-\alpha \eta^{4}\right)} \int_{0}^{1}(1-s)^{4} f(s, u(s)) d s-\frac{\alpha t^{4}}{24\left(1-\alpha \eta^{4}\right)} \int_{0}^{\eta}(\eta-s)^{4} f(s, u(s)) d s$.

By Lemma 1, the BVP (1)-(2) has a solution if and only if the operator $T$ has a fixed point in E. So we only need to seek a fixed point of $T$ in $E$. By Ascoli-Arzela theorem, we can prove that $T$ is a completely continuous operator. Now we cite the Leray-Schauder nonlinear alternative.

Lemma 2. [1]. Let $E$ be a Banach space and $\Omega$ be a bounded open subset of $E, 0 \in \Omega$. T: $\bar{\Omega} \rightarrow E$ be a completely continuous operator. Then, either

(i) there exists $u \in \partial \Omega$ and $\lambda>1$ such that $T(u)=\lambda u$, or

(ii) there exists a fixed point $u^{*} \in \bar{\Omega}$ of $T$.

\section{Existence of nontrivial solution}

In this section, we prove the existence of a nontrivial solution for the BVP (1)-(2). Suppose that $f \in$ $C([0,1] \times \mathbb{R}, \mathbb{R})$.

Theorem 3. Suppose that $f(t, 0) \neq 0, \alpha \eta^{4} \neq 1$, and there exist nonnegative functions $k, h \in L^{1}[0,1]$, such that

$$
\begin{gathered}
|f(t, x)| \leq k(t)|x|+h(t), \quad \text { a.e. }(t, x) \in[0,1] \times \mathbb{R}, \\
\left(\frac{1}{24}+\frac{1}{24\left|1-\alpha \eta^{4}\right|}\right) \int_{0}^{1}(1-s)^{4} k(s) d s+\frac{|\alpha|}{24\left|1-\alpha \eta^{4}\right|} \int_{0}^{\eta}(\eta-s)^{4} k(s) d s<1 .
\end{gathered}
$$

Then the BVP (1)-(2) has at least one nontrivial solution $u^{*} \in C[0,1]$.

Proof. Let

$$
\begin{aligned}
& M=\left(\frac{1}{24}+\frac{1}{24\left|1-\alpha \eta^{4}\right|}\right) \int_{0}^{1}(1-s)^{4} k(s) d s+\frac{|\alpha|}{24\left|1-\alpha \eta^{4}\right|} \int_{0}^{\eta}(\eta-s)^{4} k(s) d s, \\
& N=\left(\frac{1}{24}+\frac{1}{24\left|1-\alpha \eta^{4}\right|}\right) \int_{0}^{1}(1-s)^{4} h(s) d s+\frac{|\alpha|}{24\left|1-\alpha \eta^{4}\right|} \int_{0}^{\eta}(\eta-s)^{4} h(s) d s .
\end{aligned}
$$

Then $M<1$. Since $f(t, 0) \neq 0$, there exists an interval $[a, b] \subset[0,1]$ such that $\min _{a \leq t \leq b}|f(t, 0)|>0$, and as $h(t) \geq|f(t, 0)|$, a.e. $t \in[0,1]$, we have $N>0$. 
Let $A=N(1-M)^{-1}$ and $\Omega=\{u \in E:\|u\|<A\}$. Assume that $u \in \partial \Omega$ and $\lambda>1$ such that $T u=\lambda u$, then

$$
\begin{aligned}
\lambda A= & \lambda\|u\|=\|T u\|=\max _{0 \leq t \leq 1}|(T u)(t)| \\
\leq & \frac{1}{24} \max _{0 \leq t \leq 1} \int_{0}^{t}(t-s)^{4}|f(s, u(s))| d s+\max _{0 \leq t \leq 1}\left|\frac{t^{4}}{24\left(1-\alpha \eta^{4}\right)}\right| \int_{0}^{1}(1-s)^{4}|f(s, u(s))| d s \\
& +\max _{0 \leq t \leq 1}\left|\frac{\alpha t^{4}}{24\left(1-\alpha \eta^{4}\right)}\right| \int_{0}^{\eta}(\eta-s)^{4}|f(s, u(s))| d s \\
\leq & \left(\frac{1}{24}+\frac{1}{24\left|1-\alpha \eta^{4}\right|}\right) \int_{0}^{1}(1-s)^{4}|f(s, u(s))| d s+\frac{|\alpha|}{24\left|1-\alpha \eta^{4}\right|} \int_{0}^{\eta}(\eta-s)^{4}|f(s, u(s))| d s \\
\leq & \left(\frac{1}{24}+\frac{1}{24\left|1-\alpha \eta^{4}\right|}\right) \int_{0}^{1}(1-s)^{4} k(s)|u(s)| d s+\frac{|\alpha|}{24\left|1-\alpha \eta^{4}\right|} \int_{0}^{\eta}(\eta-s)^{4} k(s)|u(s)| d s \\
& +\left(\frac{1}{24}+\frac{1}{24\left|1-\alpha \eta^{4}\right|}\right) \int_{0}^{1}(1-s)^{4} h(s) d s+\frac{|\alpha|}{24\left|1-\alpha \eta^{4}\right|} \int_{0}^{\eta}(\eta-s)^{4} h(s) d s \\
= & M\|u\|+N .
\end{aligned}
$$

Therefore,

$$
\lambda \leq M+\frac{N}{A}=M+\frac{N}{N(1-M)^{-1}}=M+(1-M)=1 .
$$

This contradicts $\lambda>1$. By Lemma $2, T$ has a fixed point $u^{*} \in \bar{\Omega}$. In view of $f(t, 0) \neq 0$, the BVP (1)-(2) has a nontrivial solution $u^{*} \in E$. This completes the proof.

Theorem 4. Suppose that $f(t, 0) \neq 0, \alpha \eta^{4}<1$, and there exist nonnegative functions $k, h \in L^{1}[0,1]$, such that

$$
|f(t, x)| \leq k(t)|x|+h(t), \quad \text { a.e. }(t, x) \in[0,1] \times \mathbb{R} .
$$

If one of the following conditions holds:

1. there exists a constant $p>1$ such that

$$
\int_{0}^{1} k(s)^{p} d s<\left[\frac{24\left(1-\alpha \eta^{4}\right)(1+4 q)^{1 / q}}{2-\alpha \eta^{4}+|\alpha| \eta^{(1+4 q) / q}}\right]^{p}, \quad \frac{1}{p}+\frac{1}{q}=1 ;
$$

2. there exists a constant $\mu>-1$ such that

$$
\begin{gathered}
k(s) \leq \frac{\left(1-\alpha \eta^{4}\right)(1+\mu)(2+\mu)(3+\mu)(4+\mu)(5+\mu)}{2-\alpha \eta^{4}+|\alpha| \eta^{5+\mu}} s^{\mu}, \quad \text { a.e. } s \in[0,1], \\
\text { meas }\left\{s \in[0,1]: k(s)<\frac{\left(1-\alpha \eta^{4}\right)(1+\mu)(2+\mu)(3+\mu)(4+\mu)(5+\mu)}{2-\alpha \eta^{4}+|\alpha| \eta^{5+\mu}} s^{\mu}\right\}>0 ;
\end{gathered}
$$

3. there exists a constant $\mu>-5$ such that

$$
\begin{gathered}
k(s) \leq \frac{24\left(1-\alpha \eta^{4}\right)(5+\mu)}{2-\alpha \eta^{4}+|\alpha|}(1-s)^{\mu}, \quad \text { a.e. } s \in[0,1], \\
\text { meas }\left\{s \in[0,1]: k(s)<\frac{24\left(1-\alpha \eta^{4}\right)(5+\mu)}{2-\alpha \eta^{4}+|\alpha|}(1-s)^{\mu}\right\}>0 ;
\end{gathered}
$$

4. $k(s)$ satisfies

$$
k(s) \leq \frac{120\left(1-\alpha \eta^{4}\right)}{2-\alpha \eta^{4}+|\alpha| \eta^{5}}, \quad \text { a.e. } s \in[0,1], \text { meas }\left\{s \in[0,1]: k(s)<\frac{120\left(1-\alpha \eta^{4}\right)}{2-\alpha \eta^{4}+|\alpha| \eta^{5}}\right\}>0
$$

then the BVP (1)-(2) has at least one nontrivial solution $u^{*} \in E$. 
Proof. Let $M$ be defined as in the proof of Theorem 3. To prove Theorem 4, we only need to prove that $M<1$. Since $\alpha \eta^{4}<1$, we have

$$
\begin{aligned}
M & =\left(\frac{1}{24}+\frac{1}{24\left(1-\alpha \eta^{4}\right)}\right) \int_{0}^{1}(1-s)^{4} k(s) d s+\frac{|\alpha|}{24\left(1-\alpha \eta^{4}\right)} \int_{0}^{\eta}(\eta-s)^{4} k(s) d s \\
& =\frac{2-\alpha \eta^{4}}{24\left(1-\alpha \eta^{4}\right)} \int_{0}^{1}(1-s)^{4} k(s) d s+\frac{|\alpha|}{24\left(1-\alpha \eta^{4}\right)} \int_{0}^{\eta}(\eta-s)^{4} k(s) d s .
\end{aligned}
$$

1. Using the Hölder inequality, we have

$$
\begin{aligned}
M & \leq\left[\int_{0}^{1} k(s)^{p} d s\right]^{1 / p}\left\{\frac{2-\alpha \eta^{4}}{24\left(1-\alpha \eta^{4}\right)}\left[\int_{0}^{1}(1-s)^{4 q} d s\right]^{1 / q}+\frac{|\alpha|}{24\left(1-\alpha \eta^{4}\right)}\left[\int_{0}^{\eta}(\eta-s)^{4 q} d s\right]^{1 / q}\right\} \\
& \leq\left[\int_{0}^{1} k(s)^{p} d s\right]^{1 / p}\left[\frac{2-\alpha \eta^{4}}{24\left(1-\alpha \eta^{4}\right)}\left(\frac{1}{1+4 q}\right)^{1 / q}+\frac{|\alpha|}{24\left(1-\alpha \eta^{4}\right)}\left(\frac{\eta^{1+4 q}}{1+4 q}\right)^{1 / q}\right] \\
& <\frac{24\left(1-\alpha \eta^{4}\right)(1+4 q)^{1 / q}}{2-\alpha \eta^{4}+|\alpha| \eta^{(1+4 q) / q}} \times \frac{2-\alpha \eta^{4}+|\alpha| \eta^{(1+4 q) / q}}{24\left(1-\alpha \eta^{4}\right)(1+4 q)^{1 / q}} \\
& =1 .
\end{aligned}
$$

2. Here, we have

$$
\begin{aligned}
M< & \frac{\left(1-\alpha \eta^{4}\right)(1+\mu)(2+\mu)(3+\mu)(4+\mu)(5+\mu)}{2-\alpha \eta^{4}+|\alpha| \eta^{5+\mu}} \\
& \times\left[\frac{2-\alpha \eta^{4}}{24\left(1-\alpha \eta^{4}\right)} \int_{0}^{1}(1-s)^{4} s^{\mu} d s+\frac{|\alpha|}{24\left(1-\alpha \eta^{4}\right)} \int_{0}^{\eta}(\eta-s)^{4} s^{\mu} d s\right] \\
\leq & \frac{\left(1-\alpha \eta^{4}\right)(1+\mu)(2+\mu)(3+\mu)(4+\mu)(5+\mu)}{2-\alpha \eta^{4}+|\alpha| \eta^{5+\mu}}\left[\frac{2-\alpha \eta^{4}}{\left(1-\alpha \eta^{4}\right)} \frac{1}{(1+\mu)(2+\mu)(3+\mu)(4+\mu)(5+\mu)}\right. \\
& \left.+\frac{|\alpha|}{\left(1-\alpha \eta^{4}\right)} \frac{\eta^{5+\mu}}{(1+\mu)(2+\mu)(3+\mu)(4+\mu)(5+\mu)}\right] \\
= & \frac{\left(1-\alpha \eta^{4}\right)(1+\mu)(2+\mu)(3+\mu)(4+\mu)(5+\mu)}{2-\alpha \eta^{4}+|\alpha| \eta^{5+\mu}} \cdot \frac{2-\alpha \eta^{4}+|\alpha| \eta^{5+\mu}}{\left(1-\alpha \eta^{4}\right)(1+\mu)(2+\mu)(3+\mu)(4+\mu)(5+\mu)} \\
= & 1 .
\end{aligned}
$$

3. Here, we have

$$
\begin{aligned}
M & <\frac{24\left(1-\alpha \eta^{4}\right)(5+\mu)}{2-\alpha \eta^{4}+|\alpha|}\left[\frac{2-\alpha \eta^{4}}{24\left(1-\alpha \eta^{4}\right)} \int_{0}^{1}(1-s)^{4+\mu} d s+\frac{|\alpha|}{24\left(1-\alpha \eta^{4}\right)} \int_{0}^{\eta}(\eta-s)^{4}(1-s)^{\mu} d s\right] \\
& \leq \frac{24\left(1-\alpha \eta^{4}\right)(5+\mu)}{2-\alpha \eta^{4}+|\alpha|}\left[\frac{2-\alpha \eta^{4}}{24\left(1-\alpha \eta^{4}\right)} \int_{0}^{1}(1-s)^{4+\mu} d s+\frac{|\alpha|}{24\left(1-\alpha \eta^{4}\right)} \int_{0}^{1}(1-s)^{4+\mu} d s\right] \\
& =\frac{24\left(1-\alpha \eta^{4}\right)(5+\mu)}{2-\alpha \eta^{4}+|\alpha|}\left[\frac{2-\alpha \eta^{4}}{24\left(1-\alpha \eta^{4}\right)} \cdot \frac{1}{5+\mu}+\frac{|\alpha|}{24\left(1-\alpha \eta^{4}\right)} \cdot \frac{1}{5+\mu}\right] \\
& =\frac{24\left(1-\alpha \eta^{4}\right)(5+\mu)}{2-\alpha \eta^{4}+|\alpha|} \cdot \frac{2-\alpha \eta^{4}+|\alpha|}{24\left(1-\alpha \eta^{4}\right)(5+\mu)}=1 .
\end{aligned}
$$

4. Here, we have

$$
\begin{aligned}
M & <\frac{120\left(1-\alpha \eta^{4}\right)}{2-\alpha \eta^{4}+|\alpha| \eta^{5}}\left[\frac{2-\alpha \eta^{4}}{24\left(1-\alpha \eta^{4}\right)} \int_{0}^{1}(1-s)^{4} d s+\frac{|\alpha|}{24\left(1-\alpha \eta^{4}\right)} \int_{0}^{\eta}(\eta-s)^{4} d s\right] \\
& =\frac{120\left(1-\alpha \eta^{4}\right)}{2-\alpha \eta^{4}+|\alpha| \eta^{5}} \cdot \frac{2-\alpha \eta^{4}+|\alpha| \eta^{5}}{120\left(1-\alpha \eta^{4}\right)}=1 .
\end{aligned}
$$

This completes the proof. 
Theorem 5. Suppose that $f(t, 0) \neq 0, \alpha \eta^{4}>1$, and there exist nonnegative functions $k, h \in L^{1}[0,1]$ such that

$$
|f(t, x)| \leq k(t)|x|+h(t), \quad \text { a.e. }(t, x) \in[0,1] \times \mathbb{R} .
$$

If one of the following conditions holds:

1. there exists a constant $p>1$ such that

$$
\int_{0}^{1} k(s)^{p} d s<\left[\frac{24\left(\alpha \eta^{4}-1\right)(1+4 q)^{1 / q}}{\alpha\left(\eta^{4}+\eta^{(1+4 q) / q}\right)}\right]^{p}, \quad\left(\frac{1}{p}+\frac{1}{q}=1\right) ;
$$

2. there exists a constant $\mu>-1$ such that

$$
\begin{gathered}
k(s) \leq \frac{\left(\alpha \eta^{4}-1\right)(1+\mu)(2+\mu)(3+\mu)(4+\mu)(5+\mu)}{\alpha\left(\eta^{4}+\eta^{5+\mu}\right)} s^{\mu}, \quad \text { a.e. } s \in[0,1], \\
\operatorname{meas}\left\{s \in[0,1]: k(s)<\frac{\left(\alpha \eta^{4}-1\right)(1+\mu)(2+\mu)(3+\mu)(4+\mu)(5+\mu)}{\alpha\left(\eta^{4}+\eta^{5+\mu}\right)} s^{\mu}\right\}>0 ;
\end{gathered}
$$

3. there exists a constant $\mu>-5$ such that

$$
\begin{gathered}
k(s) \leq \frac{24\left(\alpha \eta^{4}-1\right)(5+\mu)}{\alpha\left(\eta^{4}+1\right)}(1-s)^{\mu}, \quad \text { a.e. } s \in[0,1], \\
\operatorname{meas}\left\{s \in[0,1]: k(s)<\frac{24\left(\alpha \eta^{4}-1\right)(5+\mu)}{\alpha\left(\eta^{4}+1\right)}(1-s)^{\mu}\right\}>0 ;
\end{gathered}
$$

4. $k(s)$ satisfies

$$
\begin{gathered}
k(s) \leq \frac{120\left(\alpha \eta^{4}-1\right)}{\alpha\left(\eta^{4}+\eta^{5}\right)}, \quad \text { a.e. } s \in[0,1], \\
\text { meas }\left\{s \in[0,1]: k(s)<\frac{120\left(\alpha \eta^{4}-1\right)}{\alpha\left(\eta^{4}+\eta^{5}\right)}\right\}>0,
\end{gathered}
$$

then the BVP (1)-(2) has at least one nontrivial solution $u^{*} \in E$.

Proof. Let $M$ be defined as in the proof of Theorem 3. To prove Theorem 5, we only need to prove that $M<1$. Since $\alpha \eta^{4}>1$, we have

$$
\begin{aligned}
M & =\frac{\alpha \eta^{4}}{24\left(\alpha \eta^{4}-1\right)} \int_{0}^{1}(1-s)^{4} k(s) d s+\frac{\alpha}{24\left(\alpha \eta^{4}-1\right)} \int_{0}^{\eta}(\eta-s)^{4} k(s) d s \\
& =\frac{\alpha}{24\left(\alpha \eta^{4}-1\right)}\left[\eta^{4} \int_{0}^{1}(1-s)^{4} k(s) d s+\int_{0}^{\eta}(\eta-s)^{4} k(s) d s\right] .
\end{aligned}
$$

1. Using the Hölder inequality, we have

$$
\begin{aligned}
M & \leq\left[\int_{0}^{1} k(s)^{p} d s\right]^{1 / p}\left\{\frac{\alpha \eta^{4}}{24\left(\alpha \eta^{4}-1\right)}\left[\int_{0}^{1}(1-s)^{4 q} d s\right]^{1 / q}+\frac{\alpha}{24\left(\alpha \eta^{4}-1\right)}\left[\int_{0}^{\eta}(\eta-s)^{4 q} d s\right]^{1 / q}\right\} \\
& \leq\left[\int_{0}^{1} k(s)^{p} d s\right]^{1 / p}\left[\frac{\alpha \eta^{4}}{24\left(\alpha \eta^{4}-1\right)}\left(\frac{1}{1+4 q}\right)^{1 / q}+\frac{\alpha}{24\left(\alpha \eta^{4}-1\right)}\left(\frac{\eta^{1+4 q}}{1+4 q}\right)^{1 / q}\right] \\
& <\frac{24\left(\alpha \eta^{4}-1\right)(1+4 q)^{1 / q}}{\alpha\left(\eta^{4}+\eta^{(1+4 q) / q}\right)} \times \frac{\alpha\left(\eta^{4}+\eta^{(1+4 q) / q}\right)}{24\left(\alpha \eta^{4}-1\right)(1+4 q)^{1 / q}}=1 .
\end{aligned}
$$

2. Here, we have

$$
M<\frac{\left(\alpha \eta^{4}-1\right)(1+\mu)(2+\mu)(3+\mu)(4+\mu)(5+\mu)}{\alpha\left(\eta^{4}+\eta^{5+\mu}\right)}\left[\frac{\alpha \eta^{4}}{24\left(\alpha \eta^{4}-1\right)} \int_{0}^{1}(1-s)^{4} s^{\mu} d s\right.
$$




$$
\begin{aligned}
& \left.+\frac{\alpha}{24\left(\alpha \eta^{4}-1\right)} \int_{0}^{\eta}(\eta-s)^{4} s^{\mu} d s\right] \\
\leq & \frac{\left(\alpha \eta^{4}-1\right)(1+\mu)(2+\mu)(3+\mu)(4+\mu)(5+\mu)}{\alpha\left(\eta^{4}+\eta^{5+\mu}\right)}\left[\frac{\alpha \eta^{4}}{\left(\alpha \eta^{4}-1\right)} \frac{1}{(1+\mu)(2+\mu)(3+\mu)(4+\mu)(5+\mu)}\right. \\
& \left.+\frac{\alpha}{\left(\alpha \eta^{4}-1\right)} \times \frac{\eta^{5+\mu}}{(1+\mu)(2+\mu)(3+\mu)(4+\mu)(5+\mu)}\right] \\
= & \frac{\left(\alpha \eta^{4}-1\right)(1+\mu)(2+\mu)(3+\mu)(4+\mu)(5+\mu)}{\alpha\left(\eta^{4}+\eta^{5+\mu}\right)} \cdot \frac{\alpha\left(\eta^{4}+\eta^{5+\mu}\right)}{\left(\alpha \eta^{4}-1\right)(1+\mu)(2+\mu)(3+\mu)(4+\mu)(5+\mu)} \\
= & 1 .
\end{aligned}
$$

3. Here, we have

$$
\begin{aligned}
M & <\frac{24\left(\alpha \eta^{4}-1\right)(5+\mu)}{\alpha\left(\eta^{4}+1\right)}\left[\frac{\alpha \eta^{4}}{24\left(\alpha \eta^{4}-1\right)} \int_{0}^{1}(1-s)^{4+\mu} d s+\frac{\alpha}{24\left(\alpha \eta^{4}-1\right)} \int_{0}^{\eta}(\eta-s)^{4}(1-s)^{\mu} d s\right] \\
& \leq \frac{24\left(\alpha \eta^{4}-1\right)(5+\mu)}{\alpha\left(\eta^{4}+1\right)}\left[\frac{\alpha \eta^{4}}{24\left(\alpha\left(\eta^{4}-1\right)\right.} \int_{0}^{1}(1-s)^{4+\mu} d s+\frac{\alpha}{24\left(\alpha \eta^{4}-1\right)} \int_{0}^{1}(1-s)^{4+\mu} d s\right] \\
& =\frac{24\left(\alpha \eta^{4}-1\right)(5+\mu)}{\alpha\left(\eta^{4}+1\right)}\left[\frac{\alpha \eta^{4}}{24\left(\alpha \eta^{4}-1\right)} \cdot \frac{1}{5+\mu}+\frac{\alpha}{24\left(\alpha \eta^{4}-1\right)} \cdot \frac{1}{5+\mu}\right] \\
& =\frac{24\left(\alpha \eta^{4}-1\right)(5+\mu)}{\alpha\left(\eta^{4}+1\right)} \cdot \frac{\alpha\left(\eta^{4}+1\right)}{24\left(\alpha \eta^{4}-1\right)(5+\mu)}=1 .
\end{aligned}
$$

4. Here, we have

$$
\begin{aligned}
M & <\frac{120\left(\alpha \eta^{4}-1\right)}{\alpha\left(\eta^{4}+\eta^{5}\right)}\left[\frac{\alpha \eta^{4}}{24\left(\alpha \eta^{4}-1\right)} \int_{0}^{1}(1-s)^{4} d s+\frac{\alpha}{24\left(\alpha \eta^{4}-1\right)} \int_{0}^{\eta}(\eta-s)^{4} d s\right] \\
& =\frac{120\left(\alpha \eta^{4}-1\right)}{\alpha\left(\eta^{4}+\eta^{5}\right)} \cdot \frac{\alpha\left(\eta^{4}+\eta^{5}\right)}{120\left(\alpha \eta^{4}-1\right)}=1 .
\end{aligned}
$$

This completes the proof.

Corollary 6. Suppose $f(t, 0) \neq 0, \alpha \eta^{4}<1$, and there exist nonnegative functions $k, h \in L^{1}[0,1]$ such that

$$
|f(t, x)| \leq k(t)|x|+h(t), \quad \text { a.e. }(t, x) \in[0,1] \times \mathbb{R} .
$$

If one of following conditions holds:

1. there exists a constant $p>1$ such that

$$
\int_{0}^{1} k(s)^{p} d s<\left[\frac{24\left(1-\alpha \eta^{4}\right)(1+4 q)^{1 / q}}{2-\alpha \eta^{4}+|\alpha|}\right]^{p} ; \quad \frac{1}{p}+\frac{1}{q}=1 ;
$$

2. there exists a constant $\mu>-1$ such that

$$
\begin{gathered}
k(s) \leq \frac{\left(1-\alpha \eta^{4}\right)(1+\mu)(2+\mu)(3+\mu)(4+\mu)(5+\mu)}{2-\alpha \eta^{4}+|\alpha|} s^{\mu}, \quad \text { a.e. } s \in[0,1], \\
\operatorname{meas}\left\{s \in[0,1]: k(s)<\frac{\left(1-\alpha \eta^{4}\right)(1+\mu)(2+\mu)(3+\mu)(4+\mu)(5+\mu)}{2-\alpha \eta^{4}+|\alpha|} s^{\mu}\right\}>0 ;
\end{gathered}
$$

3. $k(s)$ satisfies

$$
k(s) \leq \frac{120\left(1-\alpha \eta^{4}\right)}{2-\alpha \eta^{4}+|\alpha|}, \quad \text { a.e. } \quad s \in[0,1]
$$




$$
\text { meas }\left\{s \in[0,1]: k(s)<\frac{120\left(1-\alpha \eta^{4}\right)}{2-\alpha \eta^{4}+|\alpha|}\right\}>0,
$$

then the BVP (1)-(2) has at least one nontrivial solution $u^{*} \in E$.

Proof. We have

$$
\begin{aligned}
M & =\frac{2-\alpha \eta^{4}}{24\left(1-\alpha \eta^{4}\right)} \int_{0}^{1}(1-s)^{4} k(s) d s+\frac{|\alpha|}{24\left(1-\alpha \eta^{4}\right)} \int_{0}^{\eta}(\eta-s)^{4} k(s) d s \\
& \leq \frac{2-\alpha \eta^{4}}{24\left(1-\alpha \eta^{4}\right)} \int_{0}^{1}(1-s)^{4} k(s) d s+\frac{|\alpha|}{24\left(1-\alpha \eta^{4}\right)} \int_{0}^{1}(1-s)^{4} k(s) d s \\
& =\frac{2-\alpha \eta^{4}+|\alpha|}{24\left(1-\alpha \eta^{4}\right)} \int_{0}^{1}(1-s)^{4} k(s) d s .
\end{aligned}
$$

Proof of this corollary 6 is the same method in the proof Theorem 4. The proof is complete.

Corollary 7. Suppose that $f(t, 0) \neq 0, \alpha \eta^{4}>1$, and there exist nonnegative functions $k, h \in L^{1}[0,1]$ such that

$$
|f(t, x)| \leq k(t)|x|+h(t), \quad \text { a.e. }(t, x) \in[0,1] \times \mathbb{R} .
$$

If one of the following conditions holds:

1. there exists a constant $p>1$ such that

$$
\int_{0}^{1} k(s)^{p} d s<\left[\frac{24\left(\alpha \eta^{4}-1\right)(1+4 q)^{1 / q}}{\alpha\left(\eta^{4}+1\right)}\right]^{p} ; \quad \frac{1}{p}+\frac{1}{q}=1 ;
$$

2. there exists a constant $\mu>-1$ such that

$$
\begin{gathered}
k(s) \leq \frac{\left(\alpha \eta^{4}-1\right)(1+\mu)(2+\mu)(3+\mu)(4+\mu)(5+\mu)}{\alpha\left(\eta^{4}+1\right)} s^{\mu}, \quad \text { a.e. } s \in[0,1], \\
\text { meas }\left\{s \in[0,1]: k(s)<\frac{\left(\alpha \eta^{4}-1\right)(1+\mu)(2+\mu)(3+\mu)(4+\mu)(5+\mu)}{\alpha\left(\eta^{4}+1\right)} s^{\mu}\right\}>0 ;
\end{gathered}
$$

3. $k(s)$ satisfies

$$
\begin{gathered}
k(s) \leq \frac{120\left(\alpha \eta^{4}-1\right)}{\alpha\left(\eta^{4}+1\right)}, \text { a.e. } s \in[0,1], \\
\text { meas }\left\{s \in[0,1]: k(s)<\frac{120\left(\alpha \eta^{4}-1\right)}{\alpha\left(\eta^{4}+1\right)}\right\}>0,
\end{gathered}
$$

then the BVP (1)-(2) has at least one nontrivial solution $u^{*} \in E$.

Proof. We have

$$
\begin{aligned}
M & =\frac{\alpha \eta^{4}}{24\left(\alpha \eta^{4}-1\right)} \int_{0}^{1}(1-s)^{4} k(s) d s+\frac{\alpha}{24\left(\alpha \eta^{4}-1\right)} \int_{0}^{\eta}(\eta-s)^{4} k(s) d s \\
& \leq \frac{\alpha \eta^{4}}{24\left(\alpha \eta^{4}-1\right)} \int_{0}^{1}(1-s)^{4} k(s) d s+\frac{\alpha}{24\left(\alpha \eta^{4}-1\right)} \int_{0}^{1}(1-s)^{4} k(s) d s \\
& =\frac{\alpha\left(\eta^{4}+1\right)}{24\left(\alpha \eta^{4}-1\right)} \int_{0}^{1}(1-s)^{4} k(s) d s .
\end{aligned}
$$

The rest procedure is the same as for Theorem 5 . This completes the proof.

\section{Examples}

In order to illustrate the above results, we consider some examples. 
Example 1. Consider the following problem

$$
\begin{aligned}
& u^{(5)}+\frac{t}{3} u \sin u^{2}-\sqrt{t}+2=0, \quad 0<t<1, \\
& u(0)=0, \quad u^{\prime}(0)=u^{\prime \prime}(0)=u^{\prime \prime \prime}(0)=0, \quad u(1)=-3 u(1 / 2) .
\end{aligned}
$$

Set $\eta=1 / 2, \alpha=-3$, and

$$
\begin{gathered}
f(t, x)=\frac{t}{3} x \sin x^{2}-\sqrt{t}+2, \\
k(t)=t, \quad h(t)=\sqrt{t}+2
\end{gathered}
$$

It is easy to prove that $k, h \in L^{1}[0,1]$ are nonnegative functions, and

$$
|f(t, x)| \leq k(t)|x|+h(t), \quad \text { a.e. }(t, x) \in[0,1] \times \mathbb{R},
$$

and

$$
\alpha \eta^{4}=-\frac{3}{16} \neq 1
$$

Moreover, we have

$$
\begin{aligned}
& M=\left(\frac{1}{24}+\frac{1}{24\left|1-\alpha \eta^{4}\right|}\right) \int_{0}^{1}(1-s)^{4} k(s) d s+\frac{|\alpha|}{24\left|1-\alpha \eta^{4}\right|} \int_{0}^{\eta}(\eta-s)^{4} k(s) d s \\
& M=\frac{105}{1368} \int_{0}^{1}(1-s)^{4} s d s+\frac{6}{57} \int_{0}^{1 / 2}\left(\frac{1}{2}-s\right)^{4} s d s=\frac{105}{41040}+\frac{6}{109440}<1 .
\end{aligned}
$$

Hence, by Theorem 3, the BVP (6) has at least one nontrivial solution $u^{*}$ in $E$.

Example 2. Consider the following problem

$$
\begin{aligned}
& u^{(5)}+\frac{2 / 3 \sqrt{8+t}}{5+u^{2}} u \cos u^{3}-e^{t}+1=0, \quad 0<t<1, \\
& u(0)=0, \quad u^{\prime}(0)=u^{\prime \prime}(0)=u^{\prime \prime \prime}(0)=0, \quad u(1)=-4 u(1 / 4) .
\end{aligned}
$$

Set $\eta=1 / 4, \alpha=-4$, and

$$
\begin{gathered}
f(t, x)=\frac{2 / 3 \sqrt{8+t}}{5+x^{2}} x \cos x^{3}-e^{t}+1, \\
k(t)=\frac{2}{3} \sqrt{8+t}, \quad h(t)=e^{t}+1 .
\end{gathered}
$$

It is easy to prove that $k, h \in L^{1}[0,1]$ are nonnegative functions, and

$$
|f(t, x)| \leq k(t)|x|+h(t), \quad \text { a.e. }(t, x) \in[0,1] \times \mathbb{R} .
$$

and

$$
\alpha \eta^{4}=-\frac{1}{64}<1
$$

Let $p=q=2$, such that $\frac{1}{p}+\frac{1}{q}=1$, then

$$
\int_{0}^{1} k(s)^{p} d s=\int_{0}^{1} \frac{4}{9}(8+s) d s=\frac{34}{9} .
$$

Moreover, we have

$$
\left[\frac{24\left(1-\alpha \eta^{4}\right)(1+4 q)^{1 / q}}{2-\alpha \eta^{4}+|\alpha| \eta^{(1+4 q) / q}}\right]^{p}=1307.88
$$

Therefore,

$$
\int_{0}^{1} k(s)^{p} d s<\left[\frac{24\left(1-\alpha \eta^{4}\right)(1+4 q)^{1 / q}}{2-\alpha \eta^{4}+|\alpha| \eta^{(1+4 q) / q}}\right]^{p} .
$$


Hence, by Theorem 4(1), the BVP (7) has at least one nontrivial solution $u^{*}$ in $E$.

Example 3. Consider the following problem

$$
\begin{aligned}
& u^{(5)}+\frac{u}{7\left(4+u^{2}\right) \sqrt[3]{t}} e^{-\cos u}-2 t-1=0, \quad 0<t<1, \\
& u(0)=0, \quad u^{\prime}(0)=u^{\prime \prime}(0)=u^{\prime \prime \prime}(0)=0, \quad u(1)=-3 u(1 / 3) .
\end{aligned}
$$

Set $\eta=1 / 3, \alpha=-3$, and

$$
\begin{gathered}
f(t, x)=\frac{x}{7\left(4+x^{2}\right) \sqrt[3]{t}} e^{-\cos x}-2 t-1, \\
k(t)=\frac{1}{7 \sqrt[3]{t}}, \quad h(t)=2 t+1 .
\end{gathered}
$$

It is easy to prove that $k, h \in L^{1}[0,1]$ are nonnegative functions, and

$$
|f(t, x)| \leq k(t)|x|+h(t), \quad \text { a.e. }(t, x) \in[0,1] \times \mathbb{R} \text {. }
$$

and

$$
\alpha \eta^{4}=-\frac{1}{27}<1
$$

Let $\mu=-\frac{1}{3}>-1$, then

$$
\frac{\left(1-\alpha \eta^{4}\right)(1+\mu)(2+\mu)(3+\mu)(4+\mu)(5+\mu)}{2-\alpha \eta^{4}+|\alpha| \eta^{5+\mu}}=16.873
$$

Therefore,

$$
\begin{gathered}
k(s)=\frac{1}{7 \sqrt[3]{s}}=\frac{1}{7} s^{-\frac{1}{3}}<16.873 . s^{-\frac{1}{3}} \\
\operatorname{meas}\left\{s \in[0,1]: k(s)<\frac{\left(1-\alpha \eta^{4}\right)(1+\mu)(2+\mu)(3+\mu)(4+\mu)(5+\mu)}{2-\alpha \eta^{4}+|\alpha| \eta^{5+\mu}} s^{\mu}\right\}>0 .
\end{gathered}
$$

Hence, by Theorem 4(2), the BVP 8 has at least one nontrivial solution $u^{*}$ in $E$.

Example 4. Consider the following problem

$$
\begin{aligned}
& u^{(5)}+\frac{3 u^{3}}{7\left(1+u^{2}\right) \sqrt[3]{(1-t)^{2}}} \sin u+t^{5}-1=0, \quad 0<t<1, \\
& u(0)=0, \quad u^{\prime}(0)=u^{\prime \prime}(0)=u^{\prime \prime \prime}(0)=0, \quad u(1)=-2 u(1 / 2) .
\end{aligned}
$$

Set $\eta=1 / 2, \alpha=-2$, and

$$
\begin{gathered}
f(t, x)=\frac{3 x^{3}}{7\left(1+x^{2}\right) \sqrt[3]{(1-t)^{2}}} \sin x+t^{5}-1, \\
k(t)=\frac{3}{7 \sqrt[3]{(1-t)^{2}}}, \quad h(t)=t^{5}+1 .
\end{gathered}
$$

It is easy to prove that $k, h \in L^{1}[0,1]$ are nonnegative functions, and

$$
|f(t, x)| \leq k(t)|x|+h(t), \quad \text { a.e. }(t, x) \in[0,1] \times \mathbb{R} .
$$

and

$$
\alpha \eta^{4}=-\frac{1}{8}<1
$$


Let $\mu=-\frac{2}{3}>-4$, then

$$
\frac{24\left(1-\alpha \eta^{4}\right)(5+\mu)}{2-\alpha \eta^{4}+|\alpha|}=\frac{2808}{33}
$$

Therefore,

$$
\begin{gathered}
k(s)=\frac{3}{7 \sqrt[3]{(1-s)^{2}}}=\frac{3}{7}(1-s)^{-\frac{2}{3}}<\frac{2808}{33}(1-s)^{-\frac{2}{3}}, \\
\text { meas }\left\{s \in[0,1]: k(s)<\frac{24\left(1-\alpha \eta^{4}\right)(5+\mu)}{2-\alpha \eta^{4}+|\alpha|}(1-s)^{\mu}\right\}>0 .
\end{gathered}
$$

Hence, by Theorem 4(3), the BVP (9) has at least one nontrivial solution $u^{*}$ in $E$.

Example 5. Consider the following problem

$$
\begin{aligned}
& u^{(5)}+\frac{t u}{2\left(3+u^{2}\right)}+e^{t}-3=0, \quad 0<t<1, \\
& u(0)=0, \quad u^{\prime}(0)=u^{\prime \prime}(0)=u^{\prime \prime \prime}(0)=0, \quad u(1)=-5 u(1 / 5) .
\end{aligned}
$$

Set $\eta=1 / 5, \alpha=-5$, and

$$
\begin{gathered}
f(t, x)=\frac{t x}{2\left(3+x^{2}\right)}+e^{t}-3, \\
k(t)=\frac{t}{2}, \quad h(t)=e^{t}+3 .
\end{gathered}
$$

It is easy to prove that $k, h \in L^{1}[0,1]$ are nonnegative functions, and

$$
|f(t, x)| \leq k(t)|x|+h(t), \quad \text { a.e. }(t, x) \in[0,1] \times \mathbb{R} .
$$

and

Moreover, we have

$$
\alpha \eta^{4}=-\frac{1}{125}<1
$$

$$
\frac{120\left(1-\alpha \eta^{4}\right)}{2-\alpha \eta^{4}+|\alpha| \eta^{5}}=\frac{9450}{157}
$$

Therefore,

$$
\begin{gathered}
k(s)=\frac{s}{2}<\frac{9450}{157}, \quad s \in[0,1], \\
\text { meas }\left\{s \in[0,1]: k(s)<\frac{120\left(1-\alpha \eta^{4}\right)}{2-\alpha \eta^{4}+|\alpha| \eta^{5}}\right\}>0 .
\end{gathered}
$$

Hence, by Theorem 4(4), the BVP (10) has at least one nontrivial solution $u^{*}$ in $E$.

Acknowledgments: The authors want to thank the anonymous referee for the throughout reading of the manuscript and several suggestions that help us improve the presentation of the paper.

Author Contributions: All authors contributed equally to the writing of this paper. All authors read and approved the final manuscript.

Conflicts of Interest: "The authors declare no conflict of interest."

\section{References}

[1] Deimling, K. (1985). Nonlinear functional analysis. Springer, Berlin.

[2] Ji, Y., \& Guo, Y. (2009). The existence of countably many positive solutions for some nonlinear nth order m-point boundary value problems. Journal of Computational and Applied Mathematics, 232(2), 187-200.

[3] Graef, J. R., \& Moussaoui, T. (2009). A class of nth-order BVPs with nonlocal conditions. Computers E Mathematics with Applications, 58(8), 1662-1671.

[4] Yang, J., \& Wei, Z. (2008). Positive solutions of $n$th order m-point boundary value problem. Applied Mathematics and Computation, 202(2), 715-720. 
[5] Agarwal, R. P. (1979). Boundary Value Problems for Higher-Order Differntial Equations, MATSCIENCE. The institute of Mathematics Science. Madras-600020 (INDIA).

[6] Liu, Y., \& Ge, W. (2003). Positive solutions for $(n-1,1)$ three-point boundary value problems with coefficient that changes sign. Journal of mathematical analysis and applications, 282(2), 816-825.

[7] Eloe, P. W., \& Ahmad, B. (2005). Positive solutions of a nonlinear nth order boundary value problem with nonlocal conditions. Applied Mathematics Letters, 18(5), 521-527.

[8] Xie, D., Liu, Y., \& Bai, C. (2009). Green's function and positive solutions of a singular nth-order three-point boundary value problem on time scales. Electronic Journal of Qualitative Theory of Differential Equations, 2009(38), 1-14.

[9] Sun, Y. P. (2004). Nontrivial solution for a three-point boundary-value problem. Electronic Journal of Differential Equations, 2004(111),1-10.

[10] Sun, Y., \& Liu, L. (2004). Solvability for a nonlinear second-order three-point boundary value problem. Journal of Mathematical Analysis and Applications, 296(1), 265-275.

[11] Shuhong, L., \& Sun, Y. P. (2007). Nontrivial solution of a nonlinear second order three-point boundaryvalue problem. Applied Mathematics-A Journal of Chinese Universities Series B, 22(1), 37-47.

(C) 2019 by the authors; licensee PSRP, Lahore, Pakistan. This article is an open access article distributed under the terms and conditions of the Creative Commons Attribution (CC-BY) license (http://creativecommons.org/licenses/by/4.0/). 